\title{
NIVEL DE CONOCIMIENTO Y ACTITUD, EN ESTUDIANTES DE FORMACIÓN MAGISTERIAL, EN DOCENTES DE EDUCACIÓN BÁSICA REGULAR Y NIVEL DE VINCULACIÓN DE PADRES/MADRES O/ APODERADOS, REFERENTES A LA PERCEPCIÓN DE LAACCIÓN TUTORIAL. INSTITUCIONES EDUCATIVAS DE LA CIUDAD DE TACNA - AÑO 2009.
}

Autor: José Fernando Kong Vargas ${ }^{1}$

Universidad Privada de Tacna

E-mail: jfkong11@yahoo.com

\section{RESUMEN}

En la actualidad, a la educación se le reconoce como el factor primordial en la formación de las personas y vinculante al desarrollo de los pueblos. El profesor tutor cobra lugar de trascendental importancia para garantizar la calidad educativa; puesto que, la labor de tutoria es parte de la función docente, debiendo responder a una propuesta de educación integral, en atención a las dimensiones del desarrollo humano.

Es objetivo del presente trabajo de tesis "Comprobar la asociación entre la actitud y el conocimiento tutorial, la percepciôn de la acción tutorial de los estudiantes del ủltimo año de formación magisterial de Universidades e Institutos Superiores Pedagógicos y de los docentes de EBR. y, el nivel de vinculación de padres/madres o/apoderados con la acción tutorial en las Instituciones Educativas de la ciudad de Tacna, año 2009."

Se realizó un estudio descriptivo y causal entre los conocimientos y aplicación de la acción tutorial en una muestra de 118 estudiantes del ultimo año de Formación Docente de dos Universidades y dos Institutos Superiores Pedagógicos de la ciudad de Tacna; 434 docentes, 186 padres/madres o/apoderados de Instituciones Educativas de Educación Básica Regular

Los resultados muestran un nivel Muy Deficiente de conocimientos de tutoria en estudiantes y un nivel Deficiente en docentes. El nivel de actitud es Indiferente y Negativo en estudiantes y docentes. En ambas poblaciones muestrales, el nivel de actitud no se asocia al nivel de conocimientos. Se manifiesta, desde sus percepciones, un nivel Regular en el manejo de la acción tutorial en los estudiantes y docentes, siendo de nivel Bajo la vinculación de los padres/madres/apoderados.

Se recomienda replantear los planes de estudio de las Instituciones formadoras del magisterio, reorientar las implementaciones y capacitaciones, por parte de las autoridades educativas (Ministerio de Educación. Institutos y Universidades), teniendo en cuenta la misión tutorial que corresponde al profesor en la Institución Educativa actual; asimismo, crear estrategias que respondan a la realidad contextual de los padres/madres para involucrarlos con efectividad en la propuesta tutorial.

PALABRAS CLAVE:

Acción tutorial, actitud, conocimientos de futoria. orientación y asesoramiento.

\section{ABSTRACT}

Currently, education is recognized as a main factor in formation of people and a linker for development of nations. Tutor teachers have a trascendental importance when it comes to educational quality. Tutorial service is a part of teaching and must respond to a proposal of integral education responding to human development dimensions.

The objective of this work is to "demonstrate an association between tutorial knowledge, perception of tutorial action students of education, teachers of regular education level and level of bonding between fathers and mothers or guardians in reference to the perception of tutorial action in educationil centers in Tacna Peru 2009

This is a descriptive causal study about knowledge and futorial action in a simple of 118 students in the last year of education programs in two universities and two high level pedagogic institutes inTacna, 434 teachers, 186 fathers/mothers/guardians of regular basic education institutions were also included.

Our results show a very deficient level about tutorial work in students and a deficient level in teachers. the attitude level is indifferent and negative in both students and teachers. In both sample populations. The attitude level is not associated to the level of knowledge. A regular level is evidenced in both teachers and students about their perceptions. the level is low in linking of mothers/fathers and guardians

We recommend to remodel study plans in institutions that have education programs as well as reorient implementations and training programs in charge of authorities of the education ministry. Tutorial mission must be taken in account in the role of teachers in each institution. It is necessary to creatc also strategies that correspond to reality of fathers and mothers in order to get them involved effectively in tutorial work.

\section{KEYWORDS:}

Tutorial action, knowledge about tutorial action, orientation. Counseling.

1 MG. ENDOCENCIAUNIVERSITARIAY GESTIÓNEDUCATIVA 


\section{INTRODUCCIÓN}

Contexto:

Los países latinoamericanos atraviesan por una crisis educativa que afecta la Educación Básica Regular (niños y adolescentes): los alumnos, expuestos en un mundo cada vez más peligroso y violento, los docentes en ejercicio poco motivados a acompañar a sus alumnos en todos los aspectos de su desarrollo personal, los estudiantes de formación magisterial formados en una propuesta curricular más académica que de formación integral, los padres/madres de familia agobiados por la situación laboral, entregando menos tiempo para acompañar el desarrollo y crecimiento personal de sus hijos/hijas, las Instituciones Educativas (públicas o privadas) limitadas de recursos humanos y materiales para desarrollar planes de orientación. Pareciera que las acciones no impactan en la calidad educativa, ni en la gestión institucional y que no forman parte de una planificación y de un trabajo colectivo institucional.

La escuela actual apunta al desarrollo integral de la persona, no sólo desde el punto de vista de la adquisición de conocimientos y de técnicas, sino en el plano del desarrollo integral de la persona en todas las dimensiones del desarrollo humano, de la personalidad y de la transición al estado adulto integral, con todo lo que esto último implica, incluyendo la adaptación ocupacional, social y personal.

Comparto las declaraciones de la UNESCO que dice: "La labor de tutoría es parte de la función docente y por tanto, el profesor deberá brindar apoyo y consejo a los estudiantes para mejorar, no sólo su rendimiento académico, sino su ser mejor persona".

\section{Pregunta}

“¿Cuál es la asociación entre la actitud y el conocimiento tutorial, la percepción de la acción tutorial de los estudiantes del último año de formación magisterial de Universidades e Institutos Superiores Pedagógicos y de los docentes de EBR y el nivel de vinculación de padres/madres o/apoderados con la acción tutorial de Las Instituciones Educativas de la ciudad de Tacna, año 2009?"

\footnotetext{
Hipótesis

"No existe asociación entre el nivel de actitud y el conocimiento, la percepción la acción tutorial de los estudiantes del último año de formación magisterial de Universidades e Institutos Superiores Pedagógicos es de nivel Muy Deficiente y de los docentes de EBR es de nivel Deficiente $y$, el nivel de vinculación de padres/madres o/apoderados con la acción tutorial es Bajo, en las Instituciones Educativas de la ciudad de Tacna, año 2009".
}

\section{Justificación:}

- Conocimiento de la tutoría como función docente.

- Asociación respecto del conocimiento de tutoría y la actitud, desde la percepción de los docentes en ejercicio como de los estudiantes de formación magisterial; así mismo, de la acción tutorial y de la vinculación del padre/madre o apoderado.

- Vinculación de los principales responsables de la formación de los hijos padres/madres.

- Canalizar y favorecer el desarrollo humano integral asertivamente en los orientados.

\section{Objetivos e Hipótesis:}

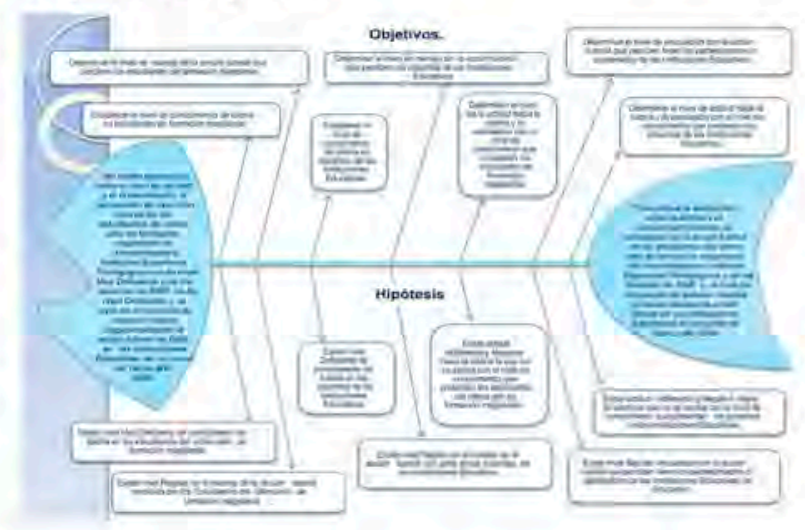

\section{Perspectiva:}

Las instituciones de Educación Superior (universitarias y no universitarias) tienen que formar a los futuros profesores en conocimiento y actitud, en el marco de la relación profesor/profesora-alumno-padre/madre; interacción que se ve fortalecida por la función que realiza el profesor a partir de la tutoría.

Los profesores en ejercicio deben tener conocimiento y actitud para saber cómo desarrollar y asumir la acción tutorial desde la misión de la educación: afectuosa, formadora, orientadora, personalizada e innovadora.

Los padres/ madres o/apoderados; deben estar involucrados en su rol e implicados en la interrelación de agentes y actores educativos para concretar la acción tutorial.

\section{Control}

Evaluar:

- A estudiantes y docentes respecto del conocimiento y la actitud en relación a la tutoría. 
La relación de dependencia entre ambos y la acción tutorial.

Conocimiento del nivel de vinculación de los padres/madres o apoderados.

Considerar: la acción tutorial desde la perspectiva de los tutorados.

Proponer: a las Instituciones Universitarias y no Universitarias de la ciudad de Tacna analizar y evaluar las propuestas curriculares que permitan la integración orgánica de la formación tutorial de los estudiantes candidatos a ser profesionales de la educación.

Ofertar: un plan de capacitación, actualización y estudios de post grado a los docentes en ejercicio que responda a la realidad contextual y atención a las "escuelas de padres".

\section{Identificación y operacionalización devariables}

Se plantean dos variables independientes: "Nivel de conocimientos de tutoría" y "Nivel de actitud respecto a la tutoria", que es el manejo de conceptos básicos y técnicas de acción tutorial; $\mathrm{y}$ la predisposición de responder de manera consciente para involucrarse en esta acción, en los estudiantes de formación magisterial y docentes del nivel primario y secundario.

Una variable dependiente: "Acción tutorial", que es una actividad orientadora, inherente a la función docente, que busca la manera específica de integrar el trabajo docente favoreciendo la individualización y la integración de los aprendizajes; así como la atención a la persona en su integridad, en consideración de "Las dimensiones humanas: corporal, afectiva, ética, espiritual, socio política, cognitiva, estética" dentro del marco de la concepción integral de la educación.

Una variable interviniente: "Vinculación del padre/madre o apoderado, donde la familia es la organización más apropiada para formar y crecer en virtudes humanas. Los padres y madres son los primeros y los más importantes maestros de sus hijos; reconocer la importancia de su papel como padre y madre tiene un efecto profundo, conducente hacia mejores logros de aprendizaje.

\section{MATERIALESY MÉTODOS}

\section{Tipo de investigación:}

El tipo de investigación es Pura, Básica o Fundamental; que tiene como objetivo conocer para mejorar los conocimientos teóricos y propiciar una actitud positiva respecto a la tutoría y orientación a los padres/madres o apoderados como base para el desarrollo integral de los alumnos en la Educación Básica Regular.

\section{Diseño de la investigación:}

El diseño adoptado es el descriptivo causal que busca caracterizar un fenómeno o hecho en base a la información recogida de varias muestras, en base a la comparación de los resultados encontrados en las mismas. En tal sentido, la presente investigación busca establecer comparaciones sobre los niveles de actitud y los niveles de conocimientos, la percepción de la acción tutorial en estudiantes y docentes y la vinculación de los/las padres/madres o apoderados en el proceso tutorial en las instituciones educativas de EBR de la ciudad Tacna-Perú.

\section{Ámbito de estudio:}

Dadas las características de la zona de estudio, se trata de un ámbito regional y específicamente se ubica en las Instituciones Educativas representativas de la zona urbana, periurbana y rural de la ciudad de Tacna.

\section{Técnicas e instrumentos:}

Para la recolección de información se empleó la tẻcnica del examen; y como instrumento, la prueba de conocimientos sobre tutoría y manejo de instrumentos de la acción tutorial.

Se aplicó también la técnica de la encuesta; utilizando como instrumento al cuestionario dirigido a los docentes de EBR y estudiantes de formación magisterial con la finalidad de identificar el conocimiento y manejo en todas las áreas de la acción tutorial. Asimismo, se aplicó un cuestionario a los padres de familia para recoger información sobre la función que están cumpliendo en la formación de sus hijos.

\section{Procesamiento y análisis de la información:}

Los resultados de la aplicación de los instrumentos se presentan en cuadros y gráficos estadísticos con descripción y análisis cualitativo y cuantitativo de los datos.

Para el tratamiento estadistico de los datos se utilizó el paquete estadistico SPSS v 15 , con las siguientes medidas:

\footnotetext{
- Medidas estadísticas de distribución normal: cálculo de frecuencias y porcentajes

- Medidas de tendencia central: cálculo de media aritmética

- Medidas de dispersión: cálculo de desviación estándar.

- La actitud se midió en escala de Likert.
}

La escala de actitud para estudiantes y docentes fue validada por alfa de Cronbach con valor alcanzado de 0.8 . 
Para la determinación de los cortes de actitud positiva, indiferente y negativa se consideraron los percentiles $25 \mathrm{y}$ 50 del puntaje total de la escala.

Para la contrastación de hipótesis se utilizó el coeficiente de correlación de Spearman y el estadístico Chi cuadrado para pruebas de bondad de ajuste. Se utilizó el valor p significativo menor a 0,05 .

Para la medición de la escala se usaron tablas de evaluación y valoración elaboradas desde la propuesta de Jorge Julio CHAVEZ PACHECO, y, Escala de Likert.

\section{RESULTADOS}

NIVEL DE CONOCIMIENTOS DE TUTORIA SEGÚN INDICADORES E INSTITUCIONES DE EDUCACIÓN SUPERIOR: ESTUDIANTES

\begin{tabular}{|c|c|c|c|c|c|c|}
\hline \multirow{2}{*}{\multicolumn{2}{|c|}{ INDICADOR }} & \multicolumn{5}{|c|}{ CENTRO DE EDUCACTON SIPERIOR } \\
\hline & & \multirow{2}{*}{\begin{tabular}{|l|} 
IPT \\
10.00 \\
\end{tabular}} & \multirow{2}{*}{$\begin{array}{c}\text { VJBG } \\
7.90 \\
\end{array}$} & \multirow{2}{*}{$\begin{array}{l}\text { AB } \\
8.10\end{array}$} & \multirow{2}{*}{\begin{tabular}{|c|} 
Eituiek \\
5,15 \\
\end{tabular}} & \multirow{2}{*}{$\begin{array}{l}\text { Total } \\
734\end{array}$} \\
\hline CONCETOS DE TUTORLA. & Medis & & & & & \\
\hline $\begin{array}{l}\text { TECNIC AS Y PROCEDIIENTOS } \\
\end{array}$ & $\begin{array}{c}\text { Desviacion } \\
\text { tipica }\end{array}$ & 6.00 & 281 & 3.30 & 3.68 & 3.40 \\
\hline \multirow{2}{*}{ MUNESO DE SITUACIONES } & Media & 16.67 & 11.95 & 1238 & 8.89 & II. A5 \\
\hline & $\begin{array}{c}\text { Desviacian } \\
\text { upica }\end{array}$ & 3.33 & 5,79 & 4.31 & 5.38 & 5,12 \\
\hline \multirow{2}{*}{$\begin{array}{l}\text { CONOCINIENTODE TEMUS } \\
\text { FORALTIVOS BASICOS }\end{array}$} & Media & 15,00 & 8.79 & 9,40 & 5,91 & 8.42 \\
\hline & $\begin{array}{c}\text { Desviación } \\
\text { tipica }\end{array}$ & 5,00 & 489 & 4.71 & 4.75 & 504 \\
\hline
\end{tabular}

NIVEL DE CONOCIMIENTOS PROMEDIO GENERAL DE TUTORIA E INSTITUCIONES DE EDUCACION SUPERIOR: ESTUDIANTES

\begin{tabular}{|l|c|c|c|c|c|}
\hline \multirow{2}{*}{$\begin{array}{c}\text { PRONIFDIO } \\
\text { GENERAL }\end{array}$} & \multicolumn{5}{|c|}{ CENTRO DE EDUCACION SUPERIOR } \\
\cline { 2 - 7 } & UPT & UJBG & JBB & Edutek & Total \\
\hline Media & 13.00 & 9.29 & 9.64 & 6.42 & 8.79 \\
\hline Desviación típica & 1.00 & 3.20 & 3.05 & 3.01 & 3.38 \\
\hline
\end{tabular}

NIVEL DE ACTITUD HACIA LA ACCIÓN TUTORIAL : ESTUDIANTES

\begin{tabular}{|c|c|c|}
\hline ACTITUD & N & $*$ \\
\hline POSITIVA & 50 & $4237 \%$ \\
\hline INDIFERENTE & 36 & $30.50 \%$ \\
\hline NEGATIVA & 32 & $27.12 \%$ \\
\hline TOTAL & 118 & T00.0\% \\
\hline
\end{tabular}

Test actitudinal, $\mathrm{P}_{25}=40, \mathrm{P}_{5 \mathrm{D}}=49$

ASOCIACIÓN ENTRE LAACTITUD Y EL NIVEL DE CONOCIMIENTO; ESTUDIANTES

\begin{tabular}{|c|c|c|c|}
\hline \multirow{2}{*}{ ACTITUD } & \multicolumn{2}{|c|}{ PROMIEDIO GENER.AL } & \multirow{2}{*}{ p } \\
\hline & Media & Desviacion tipica & \\
\hline NFGATIVA & 9.72 & 3.88 & \multirow{3}{*}{0.94} \\
\hline INDIFERENTE & 9.36 & 2.5 & \\
\hline POSITIVA & 8.04 & 3.41 & \\
\hline
\end{tabular}

NIVEL DE CONOCIMIENTOS DE TUTORIA SEGUN INDICADORES $Y$ ZONAS GEOGRÁFICAS DE UBICACION LABORAL: DOCENTES.

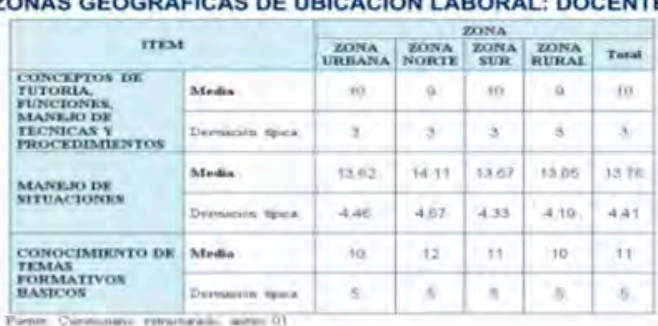

NIVEL DE CONOCIMIENTOS PROMEDIO GENERAL DE TUTORIA Y ZONAS GEOGRAFICAS DE UBICACION LABORAL: DOCENTES

\begin{tabular}{|c|c|c|c|c|c|}
\hline \multirow{2}{*}{ PROMEDIO GENERAL } & \multicolumn{5}{|c|}{ ZONA } \\
\cline { 2 - 7 } & ZONA & ZONA & ZONA & ZONA & Total \\
URBANA & NORTE & $\begin{array}{c}\text { ZUR } \\
\text { RURAL }\end{array}$ & Tot \\
\hline MEDI & 11.10 & 11.23 & 11.07 & 10.60 & 11.03 \\
\hline DESVIACION TIPICA & 3.03 & 2.93 & 2.89 & 3.08 & 2.96 \\
\hline
\end{tabular}

NIVEL DE ACTITUD HACIA LA ACCIÓN TUTORIAL : DOCENTES

\begin{tabular}{|c|c|c|}
\hline ACTITUD & $\mathrm{N}$ & 96 \\
\hline NEGATIVA & 99 & $22.8 \%$ \\
\hline DNDIFERENTE & 214 & \\
\hline POSITTVA & 121 & $27.9 \%$ \\
\hline TOTAL & 434 & $100.0 \%$ \\
\hline
\end{tabular}

ASOCIACION ENTRE LA ACTITUD Y EL NIVEL DE CONOCIMIENTO: DOCENTES

\begin{tabular}{|c|c|c|c|}
\hline \multirow{2}{*}{ ACTrID } & \multicolumn{2}{|c|}{ PROMEDIO } & \multirow{2}{*}{$\mathbf{P}$} \\
\hline & Media & Desviadén tiplea & \\
\hline NEGATTVA & 11.02 & 3.37 & \multirow{3}{*}{0.97} \\
\hline DNDHERENTI & 11.20 & 2.94 & \\
\hline positriva & 10.74 & 2.69 & \\
\hline
\end{tabular}

RESULTADO DEL TRABAJO DE CAMPO RESPECTO ALAACCION TUTORIAL EN ESTUDIANTES SEGUN INDICADORES ANALIZADOS

\begin{tabular}{|c|c|c|c|c|}
\hline $\begin{array}{l}\text { Indicaderes } \\
\text { Anabiasites }\end{array}$ & $\begin{array}{l}\text { Nivel } \\
\text { Othervado }\end{array}$ & $\begin{array}{l}\text { Sivel } \\
\text { Experade }\end{array}$ & $\begin{array}{c}\text { Valot } \\
\text { Otrsecrado }\end{array}$ & $\begin{array}{l}\text { Yolor } \\
\text { Esperado }\end{array}$ \\
\hline $\begin{array}{l}\text { Petcrpitien de } \\
\text { fonsaction } \\
\text { decrente }\end{array}$ & Bucen & Resula & 4 & 3 \\
\hline $\begin{array}{l}\text { Acinud fresue a ta } \\
\text { solacion de } \\
\text { conifirtes }\end{array}$ & Bueno & Regular & 4 & 3 \\
\hline 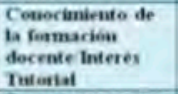 & Kegentar & Remiler & 3 & 3 \\
\hline $\begin{array}{l}\text { Fyperiencia couse } \\
\text { fatotade }\end{array}$ & Megular & Resular & 3 & 3 \\
\hline $\begin{array}{l}\text { Recoemendaciones } \\
\text { Y Propuestas para } \\
\text { b accien fed ot tal }\end{array}$ & Bucno & Regula & 4 & 3 \\
\hline
\end{tabular}


RESULTADO DEL TRABAJO DE CAMPO RESPECTO A LA ACCION SEGÚN INDICADORES ANALIZADOS

\begin{tabular}{|l|c|c|c|c|}
\hline \multicolumn{1}{|c|}{$\begin{array}{c}\text { Indicadores } \\
\text { Analizados }\end{array}$} & $\begin{array}{l}\text { Nivel } \\
\text { Observado }\end{array}$ & $\begin{array}{c}\text { Nivel } \\
\text { Esperado }\end{array}$ & $\begin{array}{c}\text { Valor } \\
\text { Observado }\end{array}$ & $\begin{array}{c}\text { Valor } \\
\text { Esperado }\end{array}$ \\
\hline $\begin{array}{l}\text { Percepcion de la } \\
\text { formaacion } \\
\text { academica }\end{array}$ & Bueno & Regular & 4 & 3 \\
\hline $\begin{array}{l}\text { Actitud frente a } \\
\text { la solucion de } \\
\text { conflictos }\end{array}$ & Bueno & Regular & 4 & 3 \\
\hline $\begin{array}{l}\text { Experiencia } \\
\text { como tutarado }\end{array}$ & Regular & Regular & 3 & 3 \\
\hline $\begin{array}{l}\text { Experiencia } \\
\text { como tutar }\end{array}$ & Bueno & Regular & 4 & 3 \\
\hline $\begin{array}{l}\text { Conocimiento de } \\
\text { orranizacion y } \\
\text { funcion tutorial }\end{array}$ & Regular & Regular & 3 & 3 \\
\hline $\begin{array}{l}\text { Recomendaciones } \\
\text { y Propuestas a ta } \\
\text { accion tutorial }\end{array}$ & Bueno & Regular & 4 & 3 \\
\hline
\end{tabular}

Fuente: Cuestionario estructurado

RESULTADO DEL TRABAJO DE CAMPO RESPECTO A LAVINCULACION EN EL PADRE / MADRE O / APODERADO EN LAACCION TUTORIAL SEGÚN INDICADORES ANALIZADOS

\begin{tabular}{|c|c|c|c|c|}
\hline $\begin{array}{l}\text { Indicadores } \\
\text { Analizados } \\
\text { Vincule familiar }\end{array}$ & $\begin{array}{l}\text { Nivel } \\
\text { Obsarvado } \\
\text { Begular }\end{array}$ & $\begin{array}{l}\text { Nivel } \\
\text { Esperade } \\
\text { Bajo }\end{array}$ & $\begin{array}{l}\text { Yalor } \\
\text { Observado } \\
3\end{array}$ & $\begin{array}{l}\text { Valar } \\
\text { Esperado }\end{array}$ \\
\hline $\begin{array}{l}\text { Interes en la } \\
\text { Formaecion del } \\
\text { purpailo }\end{array}$ & Bayo & Bayo & 2 & 2 \\
\hline 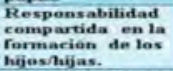 & Regular & Bajo & 3 & 2 \\
\hline 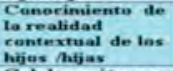 & Bape & Bayo & 2 & 2 \\
\hline $\begin{array}{l}\text { Celaboracion en } \\
\text { a rarmacion de } \\
\text { los hijos/hijas }\end{array}$ & Regular & Bajo & 3 & 2 \\
\hline $\begin{array}{l}\text { Relacion on ta } \\
\text { Aerion Tutorial }\end{array}$ & Fiegular & Bayo & 3 & 2 \\
\hline $\begin{array}{l}\text { Evaluarion, } \\
\text { wecumendarianes } \\
\text { tpropuestas a } \\
\text { Lavecion } \\
\text { tutarial. }\end{array}$ & Begular & Bajo & 3 & 2 \\
\hline
\end{tabular}

Fuente: Cuestionario estructurado

\section{Hipotesis operacional Not}

Existenivel Muy Deficientede conocimientosle tutoriaen los estudiantestel Existenivel Muy Deficientede conocimientosde tutoriaen los estudiantes uultimo ano de formación magisterial de las Univers
Superiorespedagógicosela ciudadde Tacnaaño2009

Aplicandola prueba de bondad de ajuste de los datos con Chi Cuadrado

\begin{tabular}{|c|c|}
\hline Grados de Libertad & 2 \\
\hline $\boldsymbol{\alpha}=$ & $95 \%$ \\
\hline Valor de tabla & 5.99 \\
\hline $\mathbf{X}^{2}$ & 1.16 \\
\hline
\end{tabular}

Los resultadosdemuestrarquee resultado de Chi Cuadradoal 95\% esta dentro de la zona de asm coptacionas decir el ajustede los datoses bueno, por to quela HipótesisPlanteadasaceptada

\section{Hipotesis operacional $\mathrm{N}^{202}$}

Existe nivel Deficiente de conocimientosde tutoria en los docentesde las InstitucionesEducativasde EducaciórBásicaRegulardela ciudadde Tacnaaño 2009

Aplicandola prueba de bondad de ajuste de los datos con Chi Cuadrado

\begin{tabular}{|c|c|}
\hline $\begin{array}{c}\text { Grados de } \\
\text { Libertad }\end{array}$ & 2 \\
\hline $\boldsymbol{a}=$ & $95 \%$ \\
\hline Valor de tabla & 5.99 \\
\hline $\mathbf{X}^{2}$ & 1.51 \\
\hline
\end{tabular}

Los resultadosdemuestrarque resultado de Chi Cuadrado al $95 \%$ está dentro de la zona de aceptaciónes decir el ajuste de los datoses bueno,por lo que la HipótesisPlantead saceptada

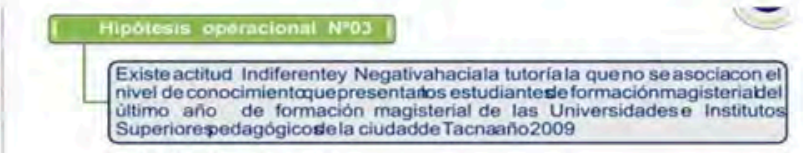
Superiorespedagógicośela ciudadde Tacnaaño 2009

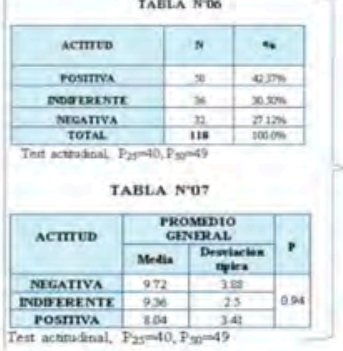

Segurios resultadom xpresadoenla tabla $\mathrm{N}^{\circ}$ 06 sólo el $4237 \%$ de los estudiantesdel uitimo ano de formación magisterialde las Universidades e Institutos Superiores pedagogicosse la ciudadde Tacnaano 2009 presentanunaactitud positivahaciala tutoria pero la cual no esta asociada aplicando expresadosen un resultadoestadisticocon (p=0.94, tabla NoO7) por lo que la hipotesis quedaceptada

\section{Hipotenis operacional NeOP

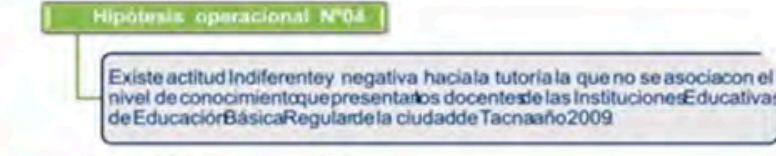 de EducaciórBásicaRegularde la cludadde Tacnaaño200}

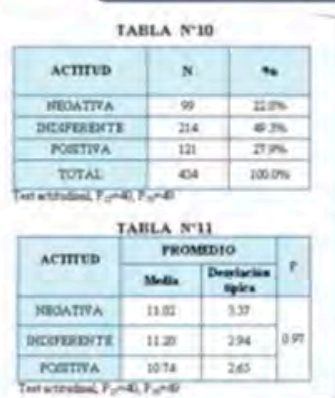

Segùnios resultadosexpresadoen la tabla No10 ef $493 \%$ de los docentes de las Institucioneszducativas de Educaciór Básica Regular de la ciudad de Tacna ano 2009 presentan una actitud indiferentey negativa hacia la tutoria pero la cual segùn prueba Spearmanno esti asociadaa su nivel de conocimientos ( $\mathrm{p}=0.97$, tabla $\mathrm{N}$ (11) on sentidoseaceptala hipotesis

\section{Hipotesis operacional No5 Existe nivel Regular en el manejo de la acción tutorial percibido por los
Estudiantes del último año de formación magisterial de las en de las
Universidadese Institutos SuperioresPedagógicosle la ciudad de Tacna año 2009}

Aplicandola prueba de bondad de ajuste de los datos con Chi Cuadrado

\begin{tabular}{|c|c|}
\hline $\begin{array}{c}\text { Grados de } \\
\text { Libertad }\end{array}$ & 4 \\
\hline $\boldsymbol{a}=$ & $95 \%$ \\
\hline Valor de Tabla & 9.49 \\
\hline $\mathbf{X}^{2}$ & 0.99 \\
\hline
\end{tabular}

El resultadodemuestraque Chi cuadradcol $95 \%$ estádentrodela zona de aceptación,es decir el ajustede los datoses muy bueno por to que la hipótesis queda aceptada

\section{Hipotesis oporacional Nob}

Existe nivel Regularen el manejo de la acción tutorial percibido por los decentes de las InstitucionesEducativasde educaciórbásicaregularciudadde Tacnaaño2009

Aplicandola prueba de bondad de ajuste de los datos con Chi Cuadrado

\begin{tabular}{|c|c|}
\hline $\begin{array}{c}\text { Grados de } \\
\text { Libertad }\end{array}$ & 5 \\
\hline $\mathbf{a}=$ & $95 \%$ \\
\hline Valor de Tabla & 11.07 \\
\hline $\mathbf{X}^{2}$ & 1.32 \\
\hline
\end{tabular}

El resultadodemuestraque $\mathrm{Chi}$ cuadradcal $95 \%$ estádentrode la zona de aceptación,es decir el ajuste de los datoses bueno la hipótesis planteada queda aceptada 


\section{Hipotesis operacional NPOT}

Existe nivel Bajo de vinculación con la acción tutorial que percibentenerlo padres/madree apoderadosle las InstitucionesEducativasde educaciórbásic regular dela ciudadde Tacnaaño2009

Aplicandola prueba de bondad de ajuste de los datos con $\mathrm{Chi}$ Cuadrado

\begin{tabular}{|c|c|}
\hline $\begin{array}{c}\text { Grados do } \\
\text { Libertad }\end{array}$ & $\boldsymbol{\sigma}$ \\
\hline $\boldsymbol{\alpha}=$ & $95 \%$ \\
\hline Valor de Tabla & 12.59 \\
\hline $\mathrm{X}^{2}$ & 1.32 \\
\hline
\end{tabular}

El resultadodemuestraque $\mathrm{Chi}$ cuadradcal 95\% estadentrodela zona de aceptación,es decir el ajuste de los datoses bueno la hipotesis planteada queda aceptada
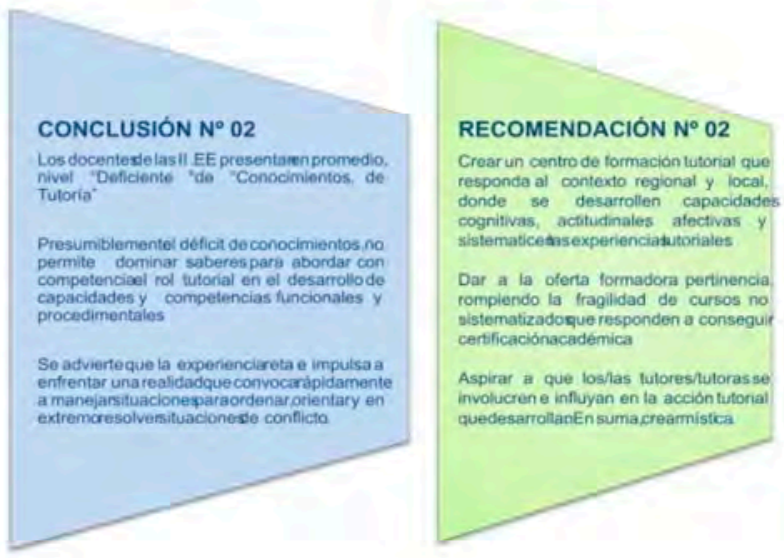

\section{Hipotosis general}

No existeasociaciórentreel nivel deactitudy el conocimientola percepciórde la accióntutorial de los estudianteslel último año de formaciónmagisterialde Universidades InstitutosSuperiore $\$$ edagógicossdenivel Muy Deficientey de los docentesde EBR es de nivel Deficientey, el nivel de vinculación de de los docentesde EBR es de nivel Deficientey, el nivel de vinculación de
padres/madreo/apoderadoson la accióntutorial es Bajo, en las Instituciones Educativasdela ciudadde Tacna año2009

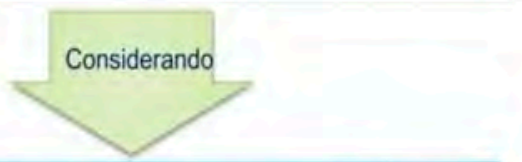

Las hipótesis operacionales anteriormentecontrastadas estadisticamentey aceptadase acuerdca los resultadosa nivel de correlación y por bondadde ajuste, podemosconcluir que la hipótesisgeneralcompuestapor las mismas, es también aceptada

\section{CONCLUSIONES Y SUGERENCIAS}
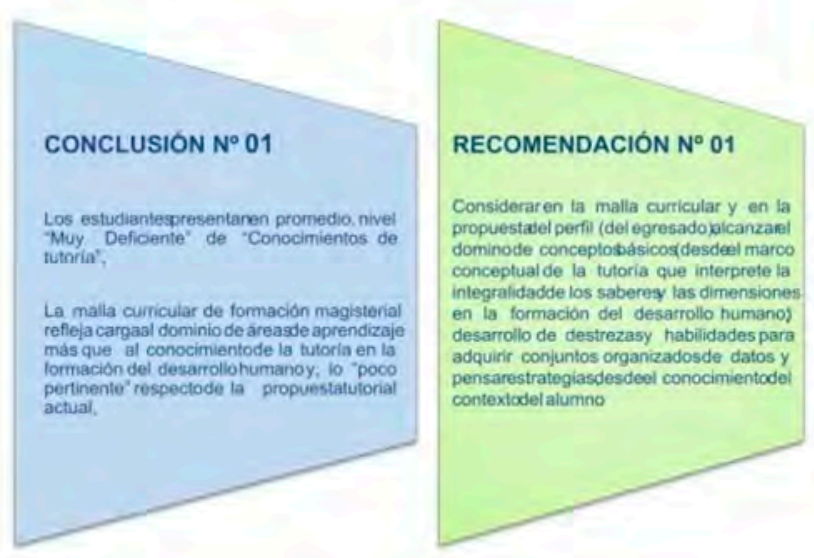
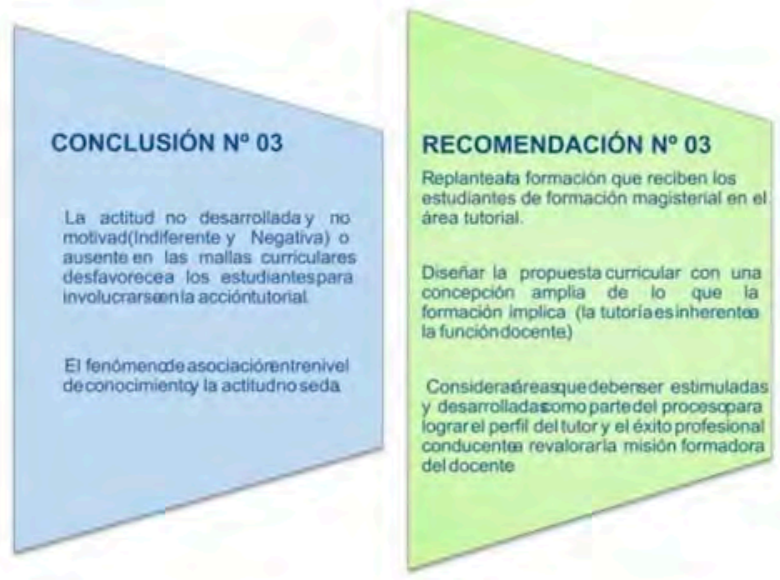
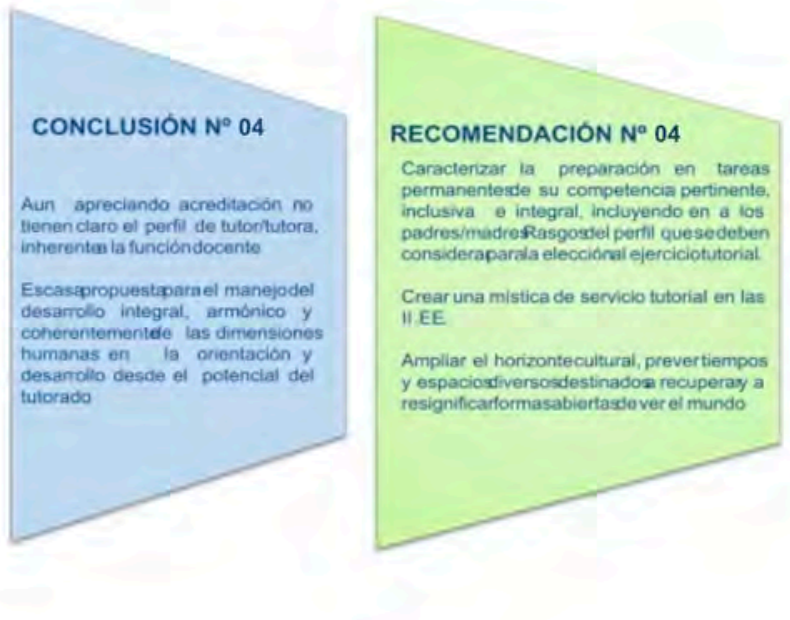


\section{REFERENCIAS}

1. DELORS, Jacques, -La Educación encierra un tesoro" (Informe de la UNESCO de la Comisión Internacional sobre la educación para el siglo XXI), Santillana, Ediciones UNESCO, 1996.

2. ACCIÓNTUTORIAL,

http://roble.pntic.mec.es/ fmoreo/acciontutorial.htm

3. Dirección de Tutoria y Orientación Educacional en la EBR. "Marco Conceptual de la Tutoría y Orientación Educacional en la EBR"-Documento de trabajoMinisterio de Educación-Perú.

4. OTUPI-Oficina de Prevención Integral "Tutoria y "Tutoria y Orientación Educativa en la educación secundaria". 2005 Ministerio de Educación República del Perú.

5. DIRECTIVAN ${ }^{\circ} 003-2005$-OTUPI/VMGP

6. CHAVEZ PACHECO, Jorge Julio. Escala de Calificaciones (Evaluación curricular) URL:

\section{Material de consulta investigativa}

7. HERNANDEZ SAMPIERI,, Roberto, et al, Metodologia de la Investigación. Santa fe de Bogotá. Editorial Me Graw-Hill, 1997.

8. SALAZAR CÓRDOVA Héctor José, "Guia de Proyecto de Investigación", CONCYTEC FENCYT, Universidad San Pedro, Escuela de Post Grado, Perú, 2008.

9. VALDIVIA DUEÑAS, M. Raủl "Elaborando la Tesis" (una propuesta) tomo I - II, Universidad Privada de Tacna, Fondo Editorial 2009.

10. VILLAMONTE, Ricardo. (2007). Metodologia de la investigación. Postgrado UNFV-Lima.

11. Metodología de la imvestigación científica. 2007. http:/www.conacyt,gov.bo/convocatorias/publicaci ones/Metodologia.pdf. 